\title{
Field Emission Properties of the Dendritic Carbon Nanotubes Film Embedded with ZnO Quantum Dots
}

\author{
Shu Zuo, Xin Li, Weihua Liu, Yongning He, Zhihao Xiao, and Changchun Zhu
}

The School of Electronic and Information Engineering, Xi'an Jiaotong University, Xi'an 710049, China

Correspondence should be addressed to Xin Li, lx@mail.xjtu.edu.cn

Received 15 April 2010; Accepted 27 June 2010

Academic Editor: Jianyu Huang

Copyright () 2011 Shu Zuo et al. This is an open access article distributed under the Creative Commons Attribution License, which permits unrestricted use, distribution, and reproduction in any medium, provided the original work is properly cited.

\begin{abstract}
Response on the effects of individual differences of common carbon nanotubes on the field emission current stability and the luminescence uniformity of cathode film, a new type of cathode film made of dendritic carbon nanotubes embedded with Zinc oxide quantum dots is proposed. The film of dendritic carbon nanotubes was synthesized through high-temperature pyrolysis of iron phthalocyanine on a silicon substrate coated with zinc oxide nanoparticles. The dendritic structure looks like many small branches protrude from the main branches in SEM and TEM images, and both the branch and the trunk are embedded with Zinc oxide quantum dots. The turn-on field of the dendritic structure film is $\sim 1.3 \mathrm{~V} / \mu \mathrm{m}$ at a current of $2 \mu \mathrm{A}$, which is much lower than that of the common carbon nanotube film, and the emission current and the luminescence uniformity are better than that of the common one. The whole film emission uniformity has been improved because the multi-emission sites out from the dendritic structure carbon nanotubes cover up the failure and defects of the single emission site.
\end{abstract}

\section{Introduction}

Carbon nanotubes have attracted wide concern due to their excellent physical, chemical, and electrical properties since they were firstly discovered by Iijima [1] in 1991. With its nanoscale emitter, high-aspect ratio, good thermal stability and conductivity carbon nanotubes are very appropriate materials for field emission. Field Emission Display based on Carbon nanotubes which have advantages in high brightness, high resolution, long life, easy working in electromagnetic radiation, and other extreme environment, is expected to be applied in the military display. However, there is still a long way for the commercial, for some critical technical problems needed to be resolved, such as emission current stability, luminescence uniformity, and long life. Some research groups had tried to improve the cathode film field emission property by optimizing the growth process of carbon nanotube on substrate [2-5]. Li research group improved contact performance by inserting a transition layer between carbon nanotubes and substrate [6-9]. Chen group $[10,11]$ discovered that the electron tunneling would be affected by the contact surface barrier between carbon nanotubes and substrate. Different Fower-Nordheim curves have been attributed for different kinds of Ohmic and Schottky contacts in their research. Shiroishi [11] research group decreased the turn-on voltage from 2.1 to $1.4 \mathrm{~V} / \mu \mathrm{m}$ and improved the field emission characteristics by laser posttreatment on the carbon nanotubes. Yu [13] group selectively etched the carbon nanotubes film prepared through screenprinting, so as to remove the inorganic binder within the CNT cathode film and to enhance emission performance effectively. A novel dendritic structure carbon nanotube cathode film was proposed in this paper. Zinc oxide nano particles film is coated on the silicon wafer through screenprinting process, which served as the growth substrate. The dendritic carbon nanotubes were then synthesized through high-temperature pyrolysis of iron phthalocyanine on the $\mathrm{ZnO}$ nanoparticle film. The dendritic carbon nanotube cathode film was embedded with $\mathrm{ZnO}$ quantum dots to bring up more emission spots. The defects in that of the single emission site type could be covered up and hided by the dendritic structure with multiemission sites, and the luminescence uniformity of the cathode film field emission could be improved. 


\section{Experiment}

In the experiment, the cathode film with dendritic structure carbon nanotubes embedded with zinc oxide quantum dots was prepared on the silicon substrates. Firstly, 10 gram ball milling zinc oxide nano particles were dispersed into $30 \mathrm{ml}$ terpineol solution. Secondly, 4 gram ethyl cellulose was dispersed in the above solution assistant with the ultrasonic so as to form the composite paste. Finally the composite paste was then heated at $200^{\circ} \mathrm{C}$. After that the zinc oxide nanoparticles film was printed on the substrate through screen-printing process. The film thickness was about 10 microns. The film was sintered in the furnace at $560^{\circ} \mathrm{C}$ to be growth substrate. Dendritic structure carbon nanotubes were grown on the composite film substrate through high-temperature pyrolysis of iron phthalocyanine chemical vapor deposition process. At last a new black film, which is about $1.5 \times 1.5$ square centimeter, was grown on the top of the substrate surface as the cathode. The schematic diagram of test setup and equivalent circuit were shown in Figure 1. The phosphor coated ITO glass is the anode that has the same size with that of the cathode. The space between the anode and the cathode is about $300 \mu \mathrm{m}$. The type of the silicon substrate is $\mathrm{N}(100)$ type that the resistivity is $10^{-2} \sim 10^{-3} \Omega . \mathrm{cm}$. The vacuum level is $1.5 \times 10^{-4} \mathrm{~Pa}$ and temperature is the room temperature of the chamber for FE measurement. Field emission characteristics of the samples had been tested with self-developed highvacuum field emission test equipment, and the luminous pictures were recorded by a digital camera.

\section{Results and Discussion}

The SEM images of the common carbon nanotube film grown on the silicon substrate and the dendritic structure carbon nanotube film grown on the $\mathrm{Si} / \mathrm{ZnO}$ composite substrate are shown in Figures 2(a) and 2(b), respectively. The two samples have been analyzed by JSM6700F scanning electron microscopy (SEM). The diameters of the carbon nanotube on the silicon substrate are from 20 to $40 \mathrm{~nm}$, and the thickness of the films is near 20 microns as shown in Figure 2(a). These carbon nanotube bundles were aligned perpendicular to the substrate in the form of high-density, and the contact between their roots and the substrate is so week that some of them are separated from the substrate. The diameter of the samples of the dendritic structure carbon nanotubes on the $\mathrm{Si} / \mathrm{ZnO}$ composite film substrate is about $100 \mathrm{~nm}$, and the thickness of dendritic structure film is about 30 to $40 \mu \mathrm{m}$ as shown in Figure 2(b), where the perspective angle is greater than 45 degrees whereas the angle from Figure 2(a) is the level perspective ( 0 degrees).

The dendritic structure carbon nanotubes are perpendicular to the substrate, and the contact between their roots and the substrate is so strong that their roots are almost completely buried in the substrate. The space between the dendritic carbon nanotubes is much larger than that of the samples of the common carbon nanotubes, which means the density of the former film is smaller than that of the latter one. As a field emission display cathode, if the space

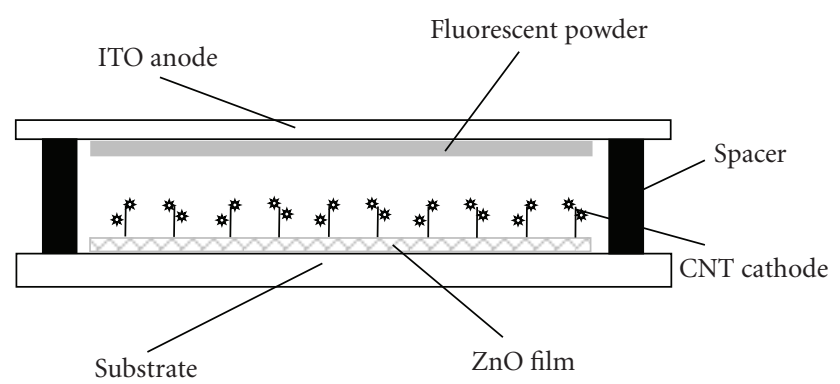

(a)

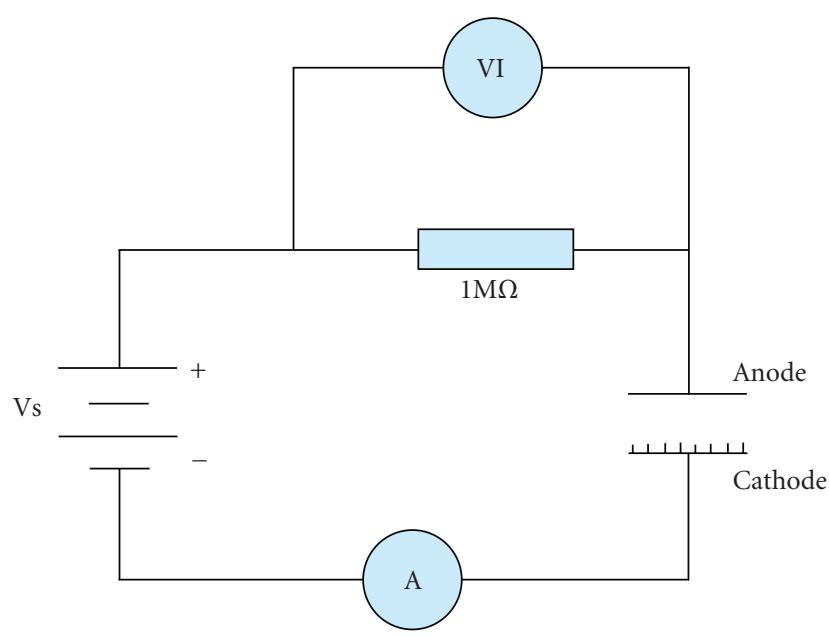

(b)

FIGURE 1: The schematic diagram (a) and equivalent circuit (b) of testing setup.

between carbon nanotubes of the film is too small to avoid shielding effect among the emission site, the emission current of the cathode film will be decreased because the local electric field of the nanotubes emission site reduced. On the other hand, keeping a certain space between emission sites will help to increase the emission current. The cathode film of the dendritic carbon nanotubes, which grow on the $\mathrm{Si} / \mathrm{ZnO}$ composite film substrate, have relative larger space in between than those grow on silicon substrate, can avoid the emission site shielding effect. When the high-emission current pass through the contact site between the roots of the each nanotube and the substrate, the contact resistances are rebuilt, changed, and become uneven because of the contact interface between of the nanotube and the substrate are uneven, so that the subsequent emission current density distribution is uneven. Because the roots of the dendritic carbon nanotubes are deeply buried in the $\mathrm{Si} / \mathrm{ZnO}$ composite film substrate, the contact resistance uniformity of each nanotube and the substrate is much better, so is the field emission current.

The higher resolution microscopic images of the dendritic carbon nanotubes are shown in Figure 3. There are many smaller nanotubes and nanoparticles covered the outer wall of the dendritic carbon nanotube. This kind of structures, which are called dendritic structure, looks like 


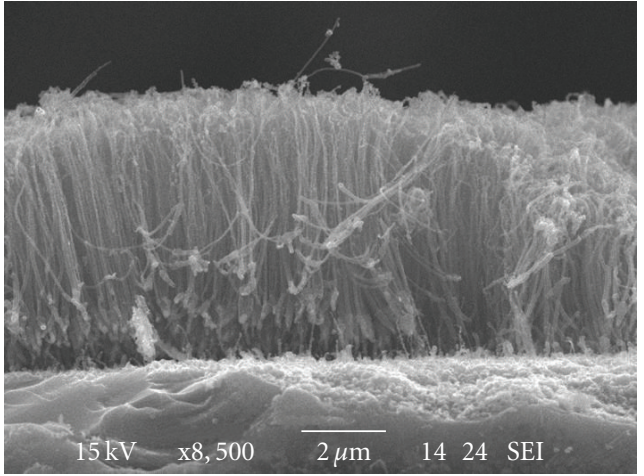

(a)

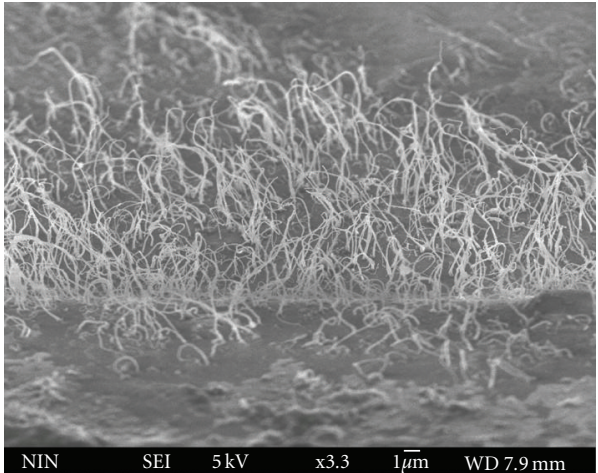

(b)

FIgURE 2: The SEM of the carbon nanotubes on (a) silicon substrates and (b) Si/ZnO substrate.

many small branches protruding from the main branches. Both the electronic transmission channels and the ratio surface area of the material increased in this kind of special dendritic structure. The nanostructure of zinc oxide particles (XRD was reported in previous work [6-9]) covered the dendritic carbon nanotube surface evenly as shown in Figures 3(a) and 3(b). The further TEM analysis also indicated that there are some zinc oxide quantum dots, which are less than $20 \mathrm{~nm}$, embedded in the nanotubes as shown in Figure 3(c). The electrons will meet the suspending $\mathrm{ZnO}$ quantum dots while they are moving along the outer layer of the dendritic carbon nanotubes from the cathode in the electric field. The electron can pass through the covalent bond between carbon nanotubes and the $\mathrm{ZnO}$ quantum dots and go into conduction band of $\mathrm{ZnO}$ quantum dots if the electric field is high enough, so that the carrier number increased a lot because of the $\mathrm{ZnO}$ quantum size effect. Both the emission current density and the field emission uniformity are improved because of the multi emission sites of the dendritic structure.

A group of field emission property tests have been carried out to know if the $\mathrm{ZnO}$ quantum dots would affect the emission current. The field emission I-V curves (Figure 4) and luminescence pictures (Figure 5) of the two samples, which are the dendritic structure carbon nanotube film 1 embedded with zinc oxide quantum dots and the common carbon nanotubes film 2 on the silicon substrate, were acquired. The vertical line corresponds to the current of $50 \mu \mathrm{A}$, and the horizontal line corresponds to voltage $500 \mathrm{~V}$ as shown in Figure 4. The turn-on voltage of the film 1 is $420 \mathrm{~V}$, and the film 2 is $749 \mathrm{~V}$. Since the space between the cathode and the anode is $300 \mu \mathrm{m}$, the turn-on field of the film 1 and film 2 are $1.3 \mathrm{~V} / \mu \mathrm{m}$ and $2.5 \mathrm{~V} / \mu \mathrm{m}$ at a current of $2 \mu \mathrm{A}$, respectively. The turn-on field of the dendritic carbon nanotubes film cathode is lower, because there is enough space between emission sites to decrease the shielding effect, and its local field of the emission site is high enough for the electronic overflow at lower voltage. The film 2 need higher electric field to produce the same emission current with that of the film 1 , for example, the voltage applied on film 1 is $1060 \mathrm{~V}$ and film 2 is $1209 \mathrm{~V}$ when the current is $100 \mu \mathrm{A}$. The electrons were transported through the carbon nanotube and the dendritic carbon nanotube embedded with zinc oxide, respectively, under the same electric field. For the latter, electrons transported through the covalent bond between the dendritic carbon nanotubes and $\mathrm{ZnO}$ quantum dots into the conduction band of zinc oxide quantum dots, cause the increasing carriers to enhance the field emission current density. Meanwhile, many new emission sites are produced by the defects that number of were formed on the surface of carbon nanotube by the zinc oxide quantum dots embedded. When the electrical field reaches turn-on field, most of the emission sites would be the current contributions. With the help of the dendritic structure carbon nanotubes embedded with zinc oxide quantum dots, the defects provoked by single site would be covered up and hided by multispot emission, so as to enhance the current and improve the emission uniformity of the whole cathode film.

Figure 5 shows the luminous pictures of the dendritic structure carbon nanotubes film cathode at different times and the same applied voltage, the emission current is about $50 \mu \mathrm{A}$. The luminescence picture of common CNTs film is shown in previous work [14]. Figure 5(a), which is the situation that just achieve this current, can be seen relatively light at lower left part, and relatively dark at the upper right corner. The pictures after 10 seconds and 20 seconds are shown in Figures 5(b) and 5(c), respectively. The initial brightness of bright areas weakened, the dark area brightness is enhanced, and the brightness of the whole region became uniform. When the $\mathrm{ZnO}$ quantum dots are embedded into the surface of carbon nanotubes, the surface of the carbon tube defects, which constitute a new emission site, each carbon nanotube is no longer a single emission site. When the electric field reach a certain value, the electrons transport into the $\mathrm{ZnO}$ conduction band through carbon nanotubes and $\mathrm{ZnO}$ quantum dots covalent bond between the quantum dots, and provoke carriers number increasing, resulting in multiemission site. The work function of $\mathrm{ZnO}$ is about $3.3 \mathrm{eV}$ that is a little lower than that of the CNT, but it is not the critical point that has improved the emission current density. The $\mathrm{ZnO}$ tip with the nano structure can emit the electrons under the lower field in previous work [15], so that the 


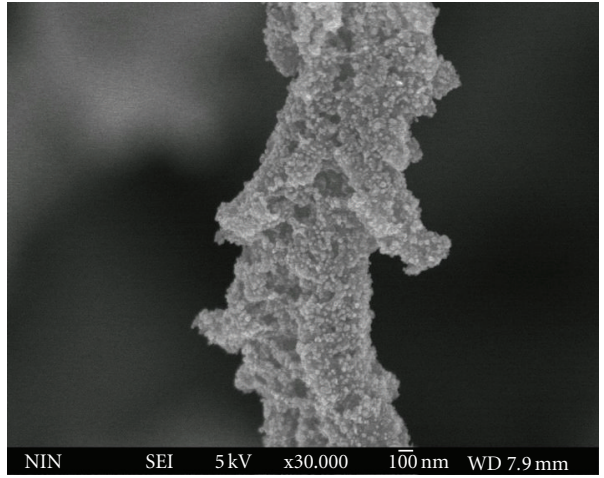

(a)

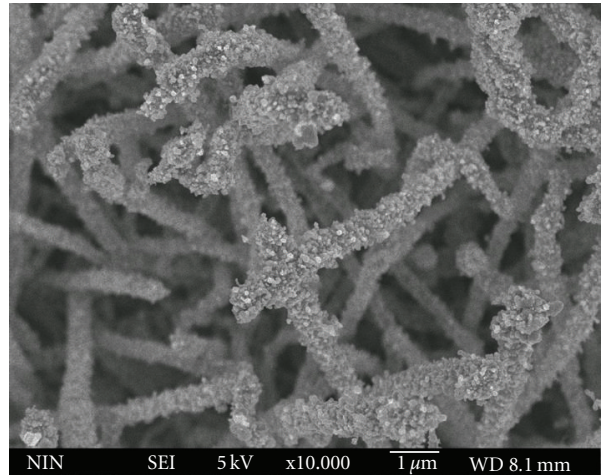

(b)

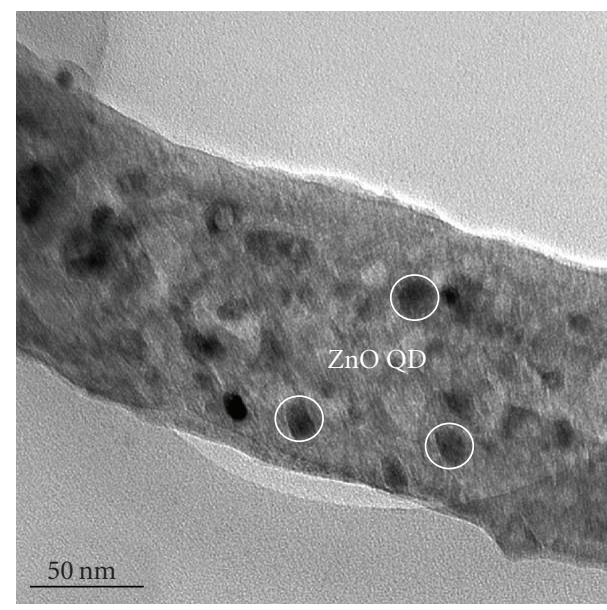

(c)

FIGURE 3: The SEM and TEM of the dendritic structure carbon nanotubes embedded with zinc oxide quantum dots. SEM of (a) one and (b) many dendritic structure carbon nanotubes; (c) TEM of the dendritic structure carbon nanotubes.

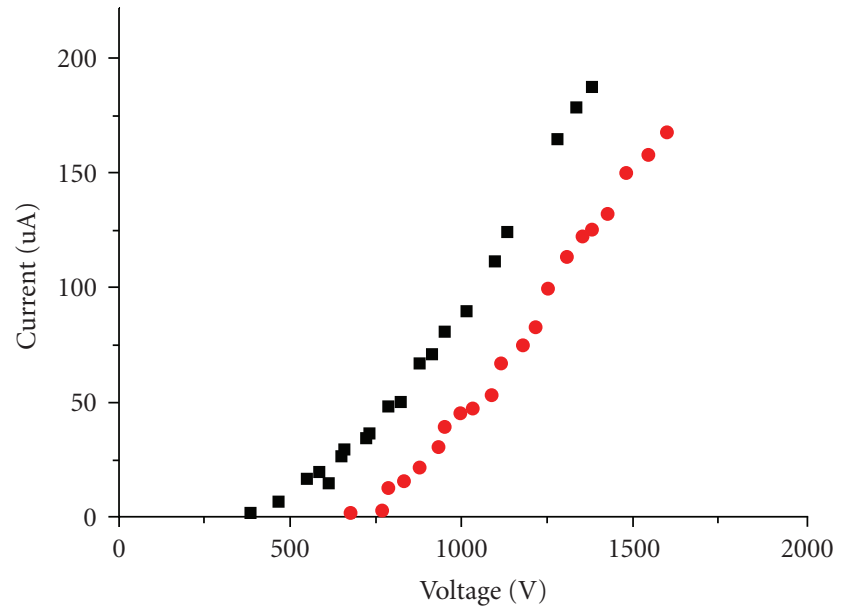

- Film 1

- Film 2

Figure 4: field emission I-V curves of samples on two different kinds of substrates. film 1: the dendritic structure carbon nanotubes embedded with zinc oxide quantum dots on the $\mathrm{ZnO}$ composite substrates; film 2: the carbon nanotubes on the silicon substrate. embedded $\mathrm{ZnO}$ QDs can indeed act as multiemission sites under this field.

Multi emission sites will cover up the failure and defects of the single site, and improve the overall emission uniformity of the cathode film. The field emission current will soon enter the saturation, and achieve field emission current stability while the applied voltage remains. The uniformity has greatly improved from the luminescence pictures.

\section{Conclusion}

Response on the effects of individual differences of common carbon nanotubes on the field emission current stability and the luminescence uniformity, zinc oxide quantum dots and nanoparticles have been proposed to modify the carbon nanotubes, so as to increase the emission sites and cover up the defects caused by initial single sites. A zinc oxide nanoparticle film was prepared on the silicon substrate through screen-printing process, the dendritic structure carbon nanotubes were synthesized on it as the cathode film. The cathode film is found to be made up of the 


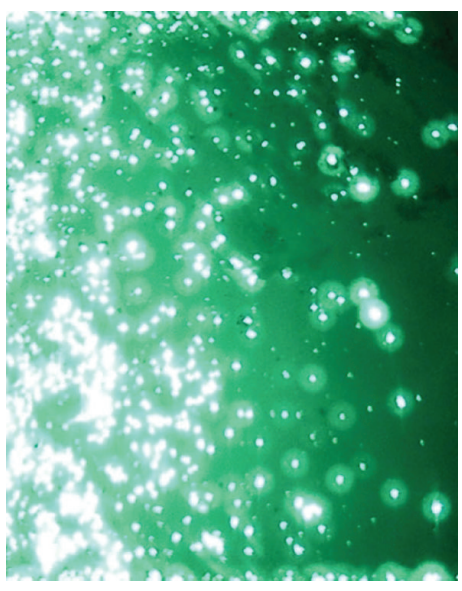

(a)

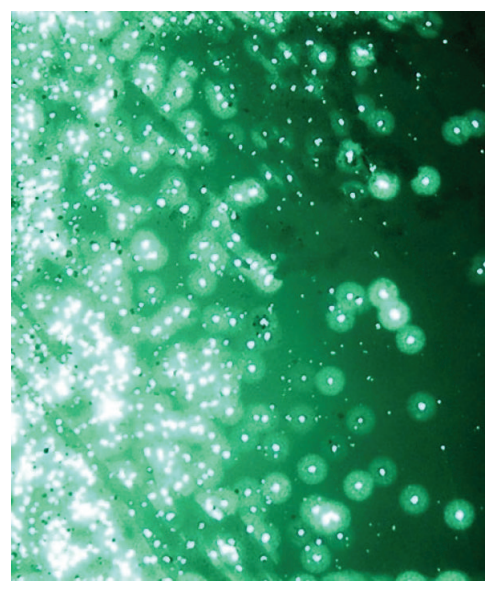

(b)

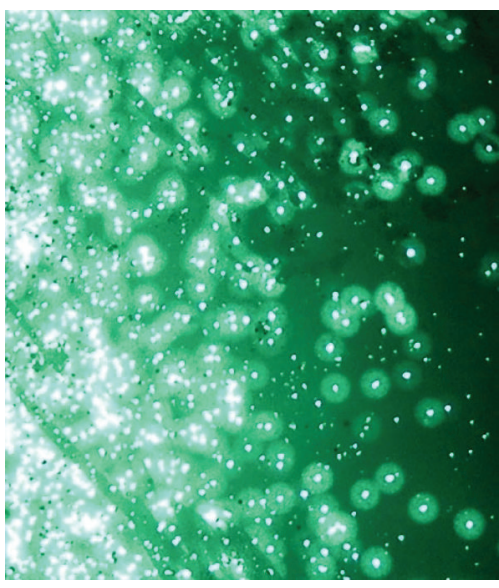

(c)

Figure 5: Field emission luminescence pictures when the emission current is $50 \mu \mathrm{A}$ : (a) just reached the current $50 \mu \mathrm{A}$; (b) after 10 second, (c) after 20 second at this current.

dendritic structure carbon nanotubes embedded with zinc oxide quantum dots, and there is enough space between emission sites to decrease the shielding effect, and its local field of the emission site is high enough for the electronic overflow at lower voltage. The contact between the root and the substrate is stronger because the roots are buried in the substrate. The turn-on field of the dendritic structure film is lower than that of the common film, and the emission current of the dendritic structure is higher than that of the common one. The additional emission sites, which are produced by the zinc oxide quantum dots embedded into the surface of carbon nanotubes, covered up the failure and defects of the single site, and improved effectively the field emission current and luminescence uniformity of the whole cathode film.

\section{Acknowledgments}

This paper was supported by the Innovation Foundation of Xi'an Applied material, Xi'an Science and Technology Program, National 863 Key Project of Panel Display, and National Science Foundation of China.

\section{References}

[1] S. Iijima, "Helical microtubules of graphitic carbon," Nature, vol. 354, no. 6348, pp. 56-58, 1991.

[2] Q. H. Wang, M. Yan, and R. P. H. Chang, "Flat panel display prototype using gated carbon nanotube field emitters," Applied Physics Letters, vol. 78, no. 9, pp. 1294-1296, 2001.

[3] J. S. Suh, K. S. Jeong, J. S. Lee, and I. Han, "Study of the fieldscreening effect of highly ordered carbon nanotube arrays," Applied Physics Letters, vol. 80, no. 13, 2002.

[4] S. H. Jo, Y. Tu, Z. P. Huang, D. L. Carnahan, D. Z. Wang, and Z. F. Ren, "Effect of length and spacing of vertically aligned carbon nanotubes on field emission properties," Applied Physics Letters, vol. 82, no. 20, pp. 3520-3522, 2003.

[5] S. Y. Lee, W. C. Choi, C. Jeon, C.-Y. Park, J. H. Yang, and M. H. Kwon, "Field emitter density control effect on emission current density by Ag-Cu alloy coating on carbon nanotubes," Applied Physics Letters, vol. 93, no. 10, Article ID 103101, 2008.

[6] X. Li, F. Ding, W. Liu, Y. He, and C. Zhu, "Luminescence uniformity studies on dendrite bamboo carbon submicrontube field-emitter arrays," Journal of Vacuum Science and Technology B, vol. 26, no. 1, pp. 171-174, 2008.

[7] J. Y. Pan, C. C. Zhu, and Y. L. Gao, "Enhanced field emission characteristics of zinc oxide mixed carbon nano-tubes films," Applied Surface Science, vol. 254, no. 13, pp. 3787-3792, 2008.

[8] Y. X. Liu, J. H. Liu, and C. C. Zhu, "Flame synthesis of carbon nanotubes for panel field emission lamp," Applied Surface Science, vol. 255, no. 18, pp. 7985-7989, 2009.

[9] L.-M. Yu and C.-C. Zhu, "Field emission characteristics study for $\mathrm{ZnO} / \mathrm{Ag}$ and $\mathrm{ZnO} / \mathrm{CNT}$ s composites produced by DC electrophoresis," Applied Surface Science, vol. 255, no. 20, pp. 8359-8362, 2009.

[10] H. F. Chen, H. Song, L. Z. Cao, et al., "Effect of intergace barrier between carbon nanotube film and substrate on field emission," Applied Physics, vol. 106, Article ID 033703, 2009.

[11] T. Shiroishi, A. Hosono, A. Sono et al., "Improvement of emission characteristics uniformity of carbon nanotube field emission display by surface treatment," Journal of Vacuum Science and Technology B, vol. 24, no. 2, pp. 979-982, 2006.

[12] K. Ohsumi, T. Honda, W. S. Kim et al., "KrF laser surface treatment of carbon nanotube cathodes with and without reactive ion etching," Journal of Vacuum Science and Technology B, vol. 25, no. 2, pp. 557-560, 2007.

[13] J. Yu, J. Chen, S. Z. Deng, J. C. She, and N. S. Xu, "Posttreatment of screen-printed carbon nanotube emitter by selective plasma etching," Journal of Vacuum Science and Technology B, vol. 25, no. 2, pp. 552-556, 2007.

[14] W. Liu, F. Zeng, L. Xin, C. Zhu, and Y. He, “Turn-on field distribution of field-emitting sites in carbon nanotube film: study with luminescent image," Journal of Vacuum Science and Technology B, vol. 26, no. 1, pp. 32-35, 2008.

[15] L.-M. Yu and C.-C. Zhu, "Electrophoresis deposition and field emission properties of composites films $\mathrm{ZnO}$ nanoneedles/CNTs," in Proceedings of the 4th IEEE International Conference on Nano/Micro Engineered and Molecular Systems (NEMS '09), pp. 9-12, Shenzhen, China, January 2009. 

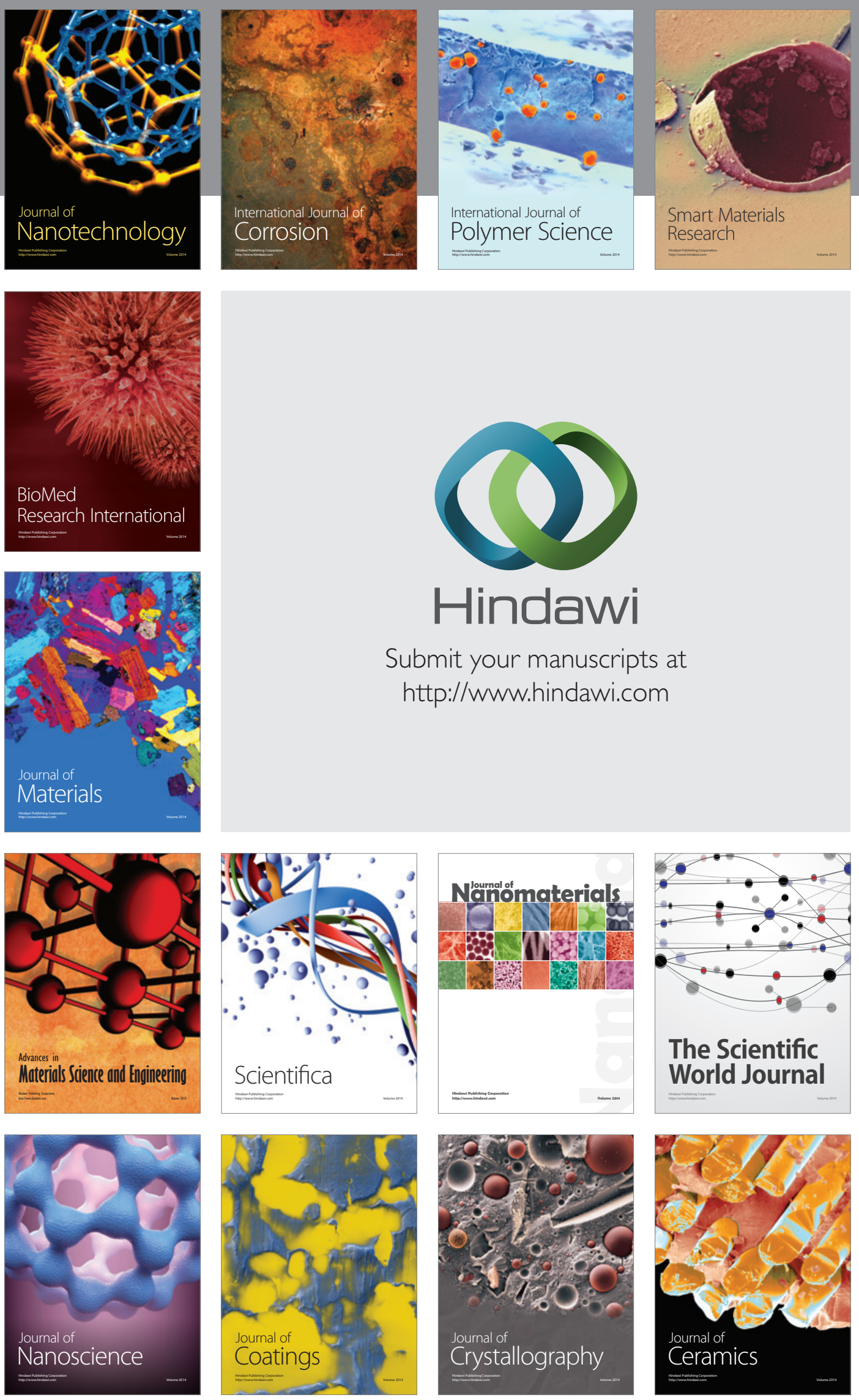

The Scientific World Journal

Submit your manuscripts at

http://www.hindawi.com

\section{World Journal}

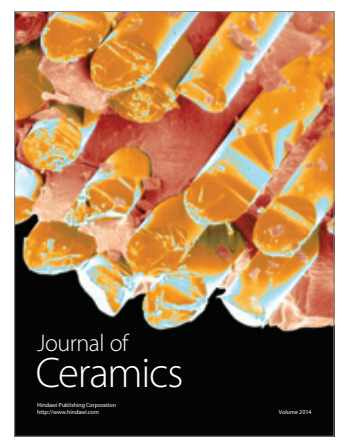

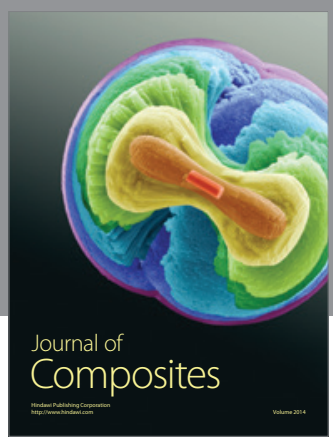
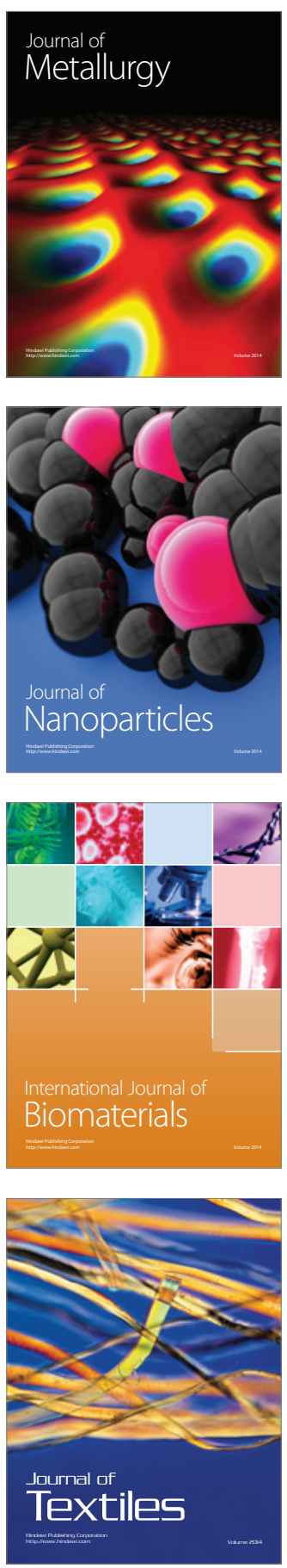